\title{
Pristine Polysulfone Networks as a Class of Polysulfide- Derived High-Performance Functional Materials
}

Maciej Podgórski, ${ }^{\mathrm{a}, \mathrm{b}}$ Chen Wang, ${ }^{\mathrm{a}}$ Ye Yuan, ${ }^{\mathrm{c}}$ Danielle Konetski, ${ }^{\mathrm{a}}$ Ivan Smalyukh ${ }^{\mathrm{c}}$ and Christopher N. Bowman ${ }^{\mathrm{a} *}$

${ }^{a}$ Department of Chemical and Biological Engineering, University of Colorado, 3415 Colorado Ave, JSC Biotech Building, Boulder, CO 80309, USA

${ }^{\mathrm{b}}$ Department of Polymer Chemistry, Faculty of Chemistry, Maria Curie-Sklodowska University, Gliniana St. 33, 20-614 Lublin, Poland

${ }^{\mathrm{c}}$ Department of Physics, University of Colorado, 2000 Colorado Ave, Boulder, CO 80309, USA

E-mail: maciej.podgorski@colorado.edu, christopher.bowman@ colorado.edu

(a) Oxidation of dipropyl sulfide with $30 \%$ hydrogen peroxide in methanol (50/50 vol. \%)

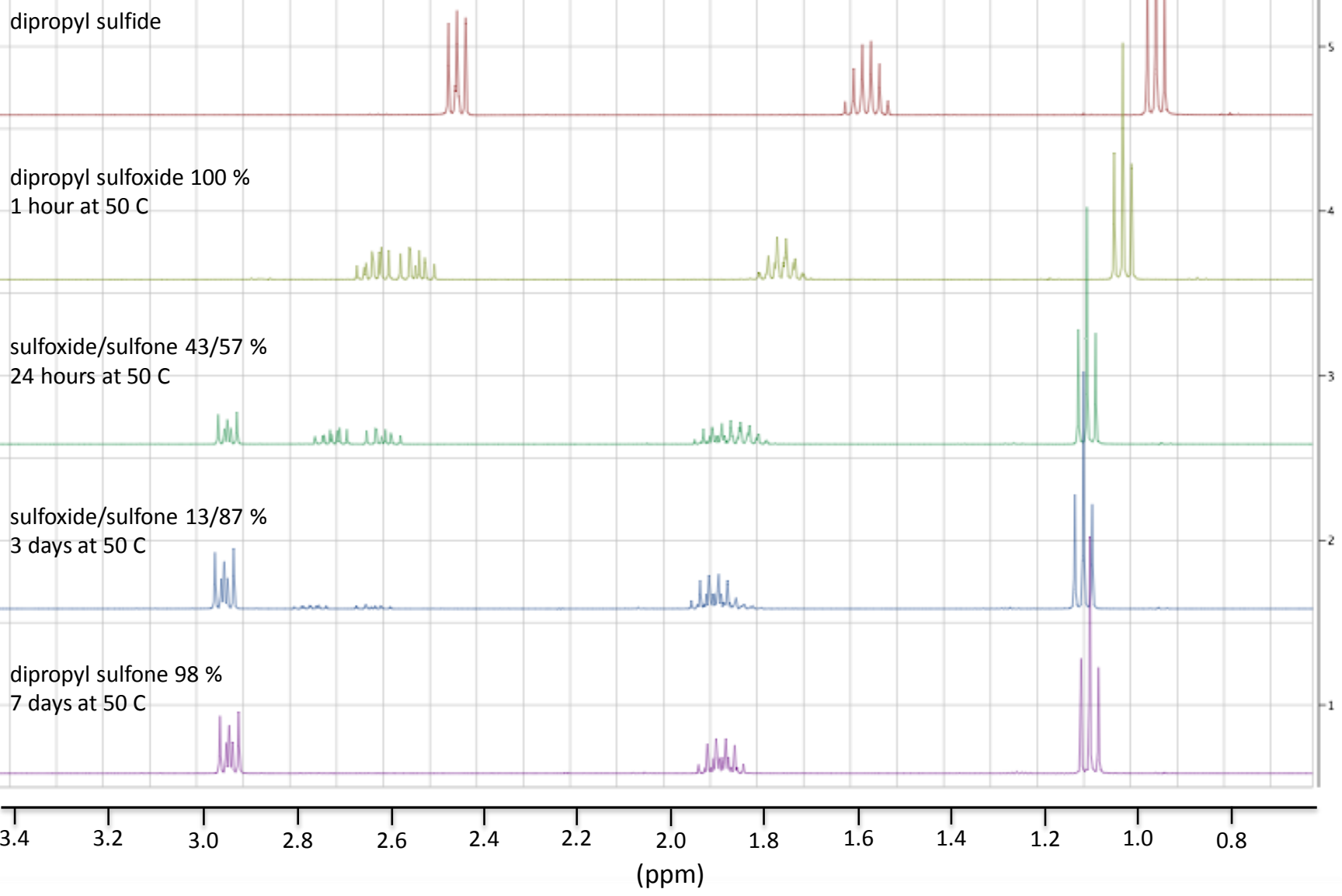


(b) Oxidation of dibutyl sulfide with $30 \%$ hydrogen peroxide in methanol (50/50 vol. \%)

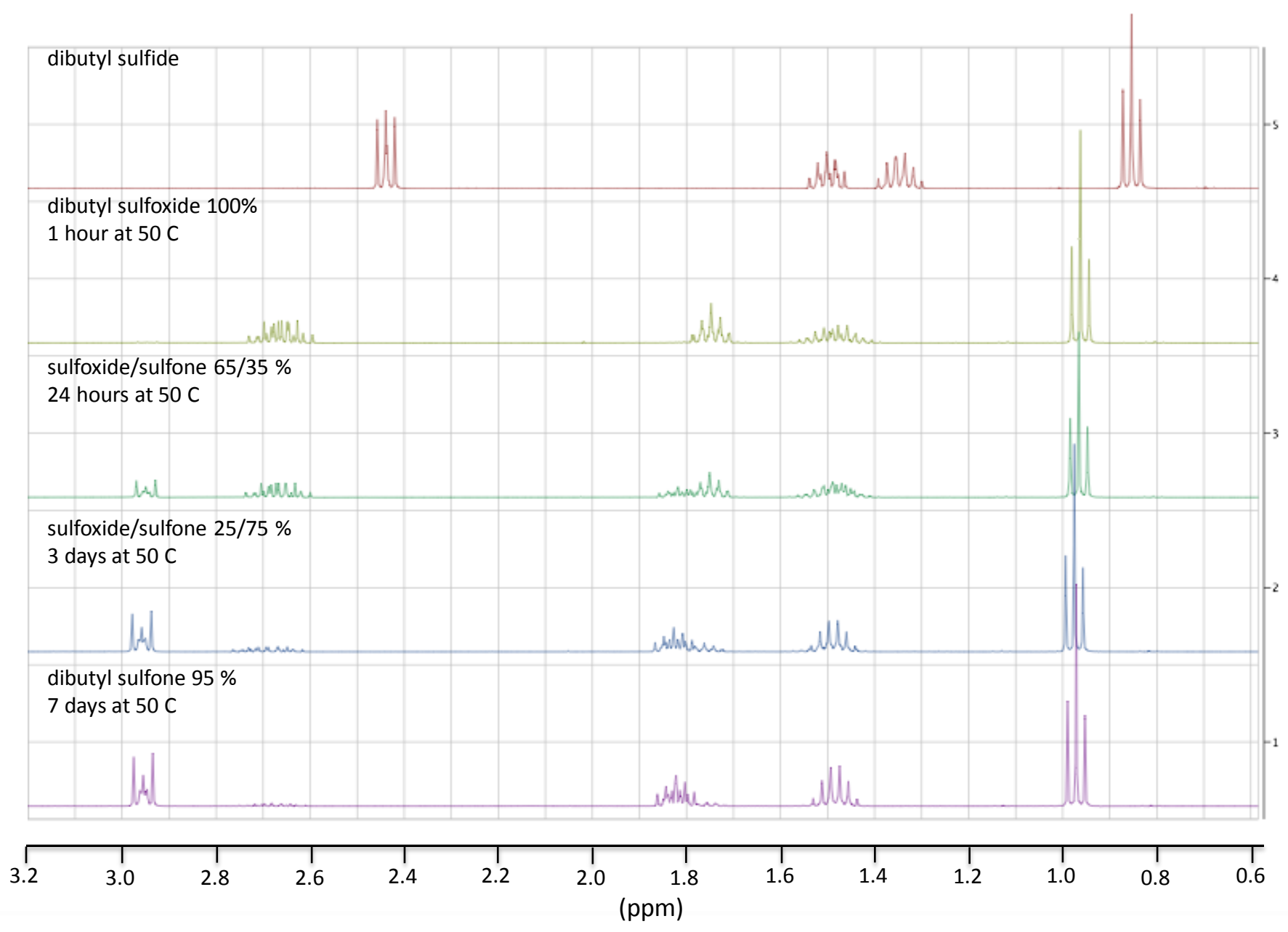

Figure $\mathrm{S} 1 . \mathrm{H}^{1} \mathrm{NMR}$ spectra of intermediate products (sulfoxides and sulfones) of dipropyl oxidation (a) and dibutyl oxidation (b). 
(a)

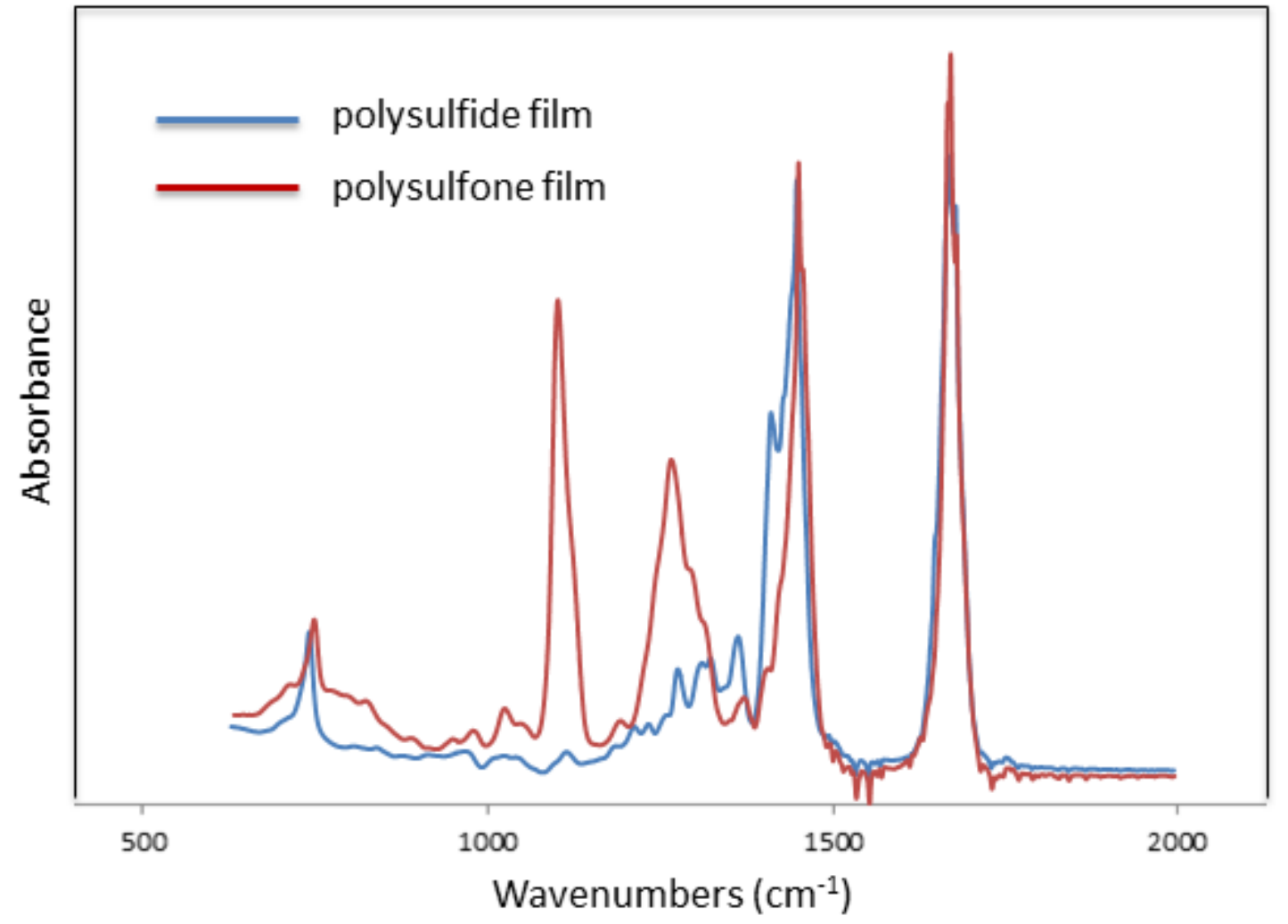

(b)

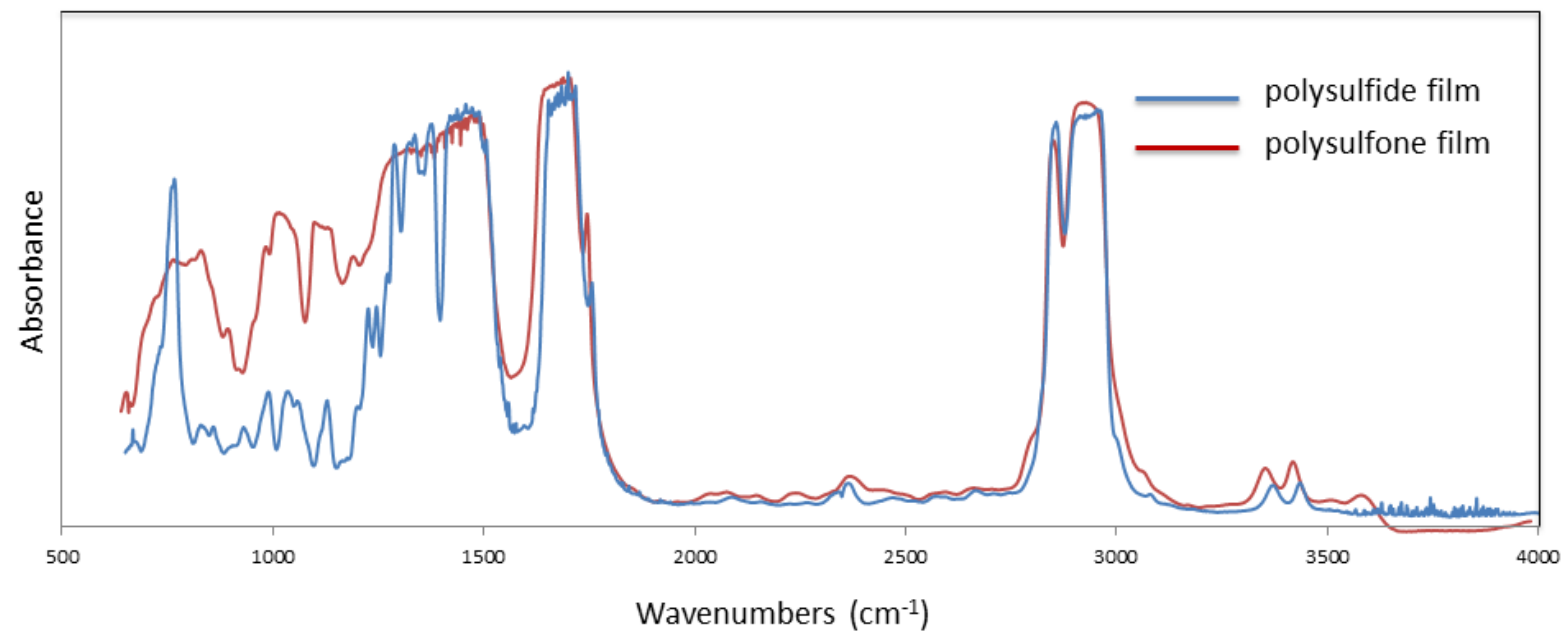

Figure S2. ATR (a) and transmission IR (b) of polysulfide and polysulfone networks. Evident are strong sulfone absorption bands in the range of $1080-1160 \mathrm{~cm}^{-1}\left(\max\right.$. at $\left.1110 \mathrm{~cm}^{-1}\right)$ and $1230-1340 \mathrm{~cm}^{-1}$ (max. at $1300 \mathrm{~cm}^{-1}$ ). 
(a)

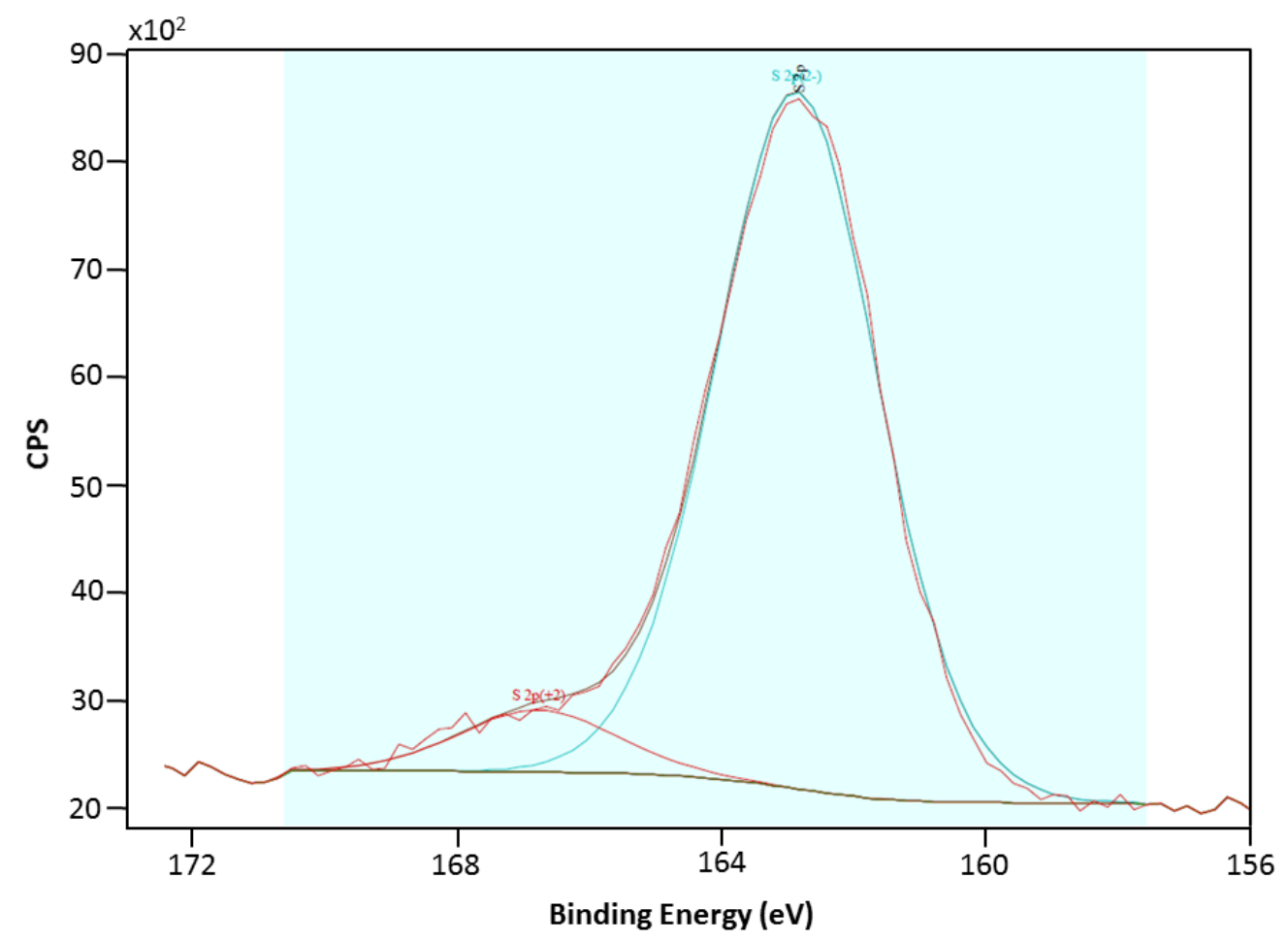

(b)

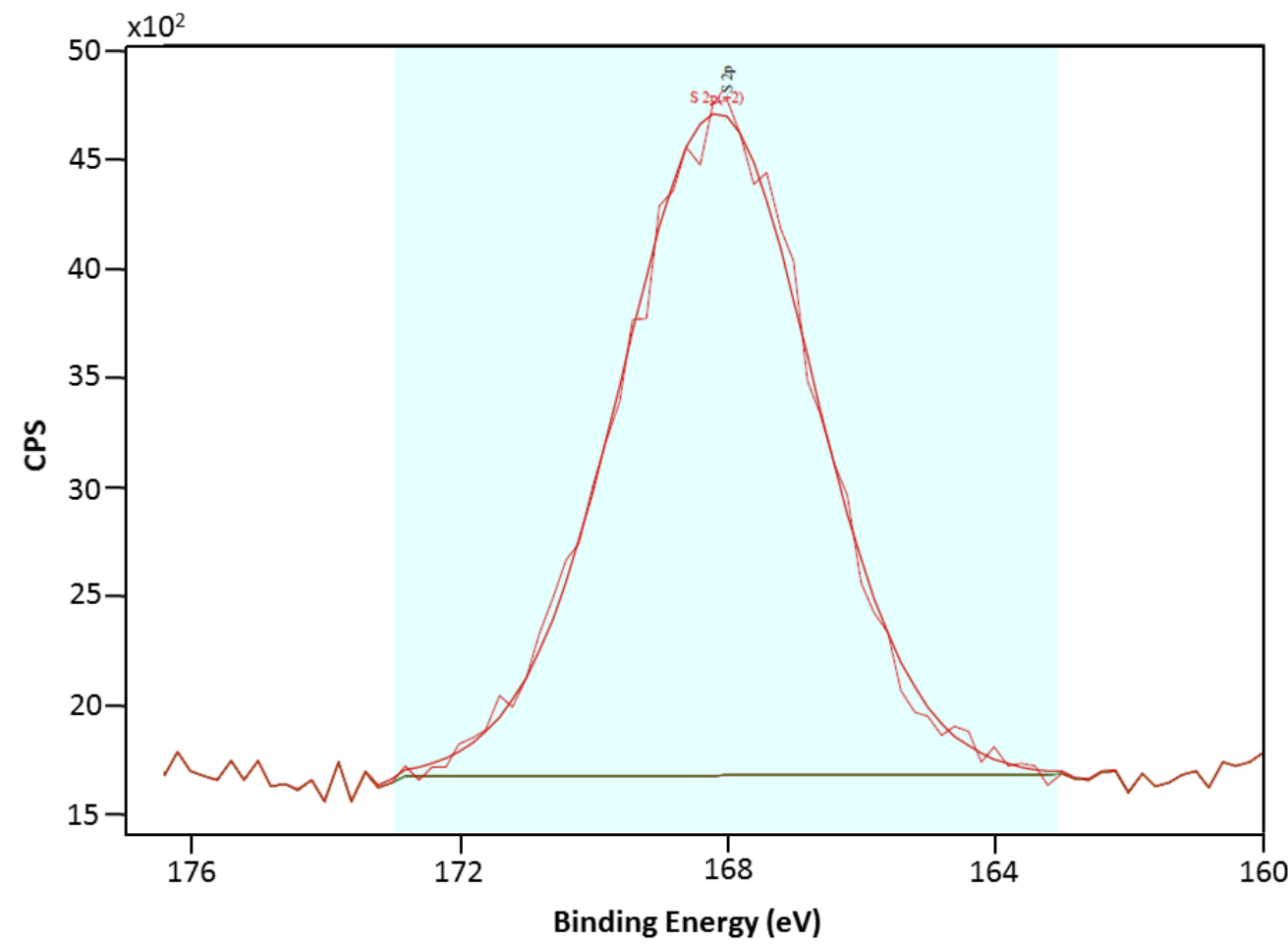

Figure S3. X-ray photoelectron spectroscopy (XPS) identification of sulfur in HDT/TTT thiol-ene film (a) and oxidized HDT/TTT sample (b). Sulfide binding energy in polysulfide material was found to be equal to $163 \mathrm{eV}$. There is also around $8 \%$ of total amount of sulfur 
in higher oxidation state $(167 \mathrm{eV})$, designated as $\mathrm{S}(+2)$ although it could be $\mathrm{S}(0)$, indicative of sulfoxides. After oxidation the binding energy shifts to $168 \mathrm{eV}$, and is presumed to be related with sulfone $S(+2)$.

Thiol-Michael particle synthesis, oxidation and analysis:

In a round bottom flask, to $50 \mathrm{~mL}$ of methanol trimethylolpropane tris(3-mercaptopropionate) ( $1.37 \mathrm{~g}, 10 \mathrm{mmol}$ of thiol group), divinyl sulfone ( $0.63 \mathrm{~g}, 10 \mathrm{mmol}$ of vinyl group) and polyvinylpyrrolidone (mol wt. 40000) ( $0.4 \mathrm{~g}, 20 \mathrm{wt} \%$ to monomers) was dissolved and a clear colorless solution was obtained. Under $400 \mathrm{rpm}$ mechanical overhead stirring, triethylamine $(0.1 \mathrm{~g}, 5 \mathrm{wt} \%$ to monomers) was added and the reaction mixture turned turbid immediately. The reaction was continued under stirring for $1 \mathrm{~h}$.

For oxidation, $5 \mathrm{~mL}$ of hydrogen peroxide was added to $5 \mathrm{~mL}$ of particle dispersion in methanol and the mixture was stirred overnight. Before characterization of both unoxidized and oxidized samples, the particles were washed with methanol for three times.

Differential scanning calorimetry (DSC)

The glass transition temperature of microparticles were determined by DSC. On a TA Q2000, $5 \mathrm{mg}$ of dried microparticles were scanning against nitrogen at a heating rate of $10^{\circ} \mathrm{C} / \mathrm{min}$. The glass transition temperature was determined from the second heating run.

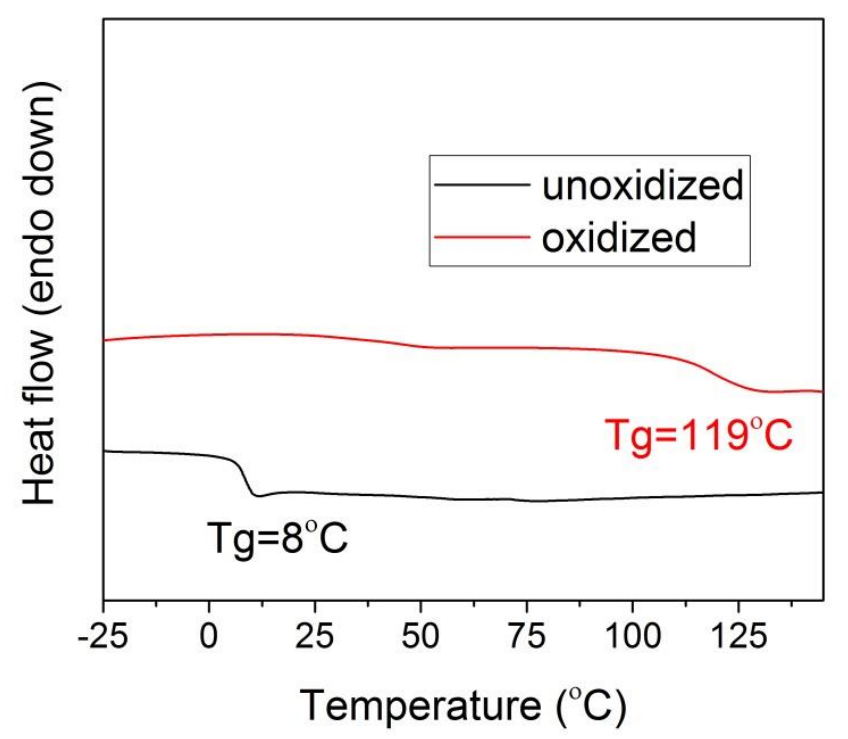

Figure S4. DSC plots for unoxidized and oxidized thiol-Michael microparticles.

Scanning electron microscopy (SEM)

SEM images were obtained on a JEOL JSM 7401F FE-SEM. Unoxidized microsparticles were diluted and re-dispersed in methanol, while oxidized microparticles were re-dispersed in water. Particle dispersions were dropped onto clean glass slide and subsequently dried. The 
size and size distribution was measured from SEM images and at least 50 individual microspheres were measured for each sample.

\section{Nanoindentation tests:}

Material: Oxidized and unoxidized polymer buffaloes (TMPTMP/TTT) printed on glass

Tip: $5 \mu \mathrm{m}$ radius spherical tip, calibrated on silica

Transducer: $1215-221$

Load function: Unoxidized: 30 second load to $150 \mathrm{~nm}, 2$ second hold at $150 \mathrm{~nm}, 30$ second unload. Oxidized: 30 second load to $150 \mathrm{~nm}, 120$ second hold at $150 \mathrm{~nm}, 30$ second unload.

Indents: 26 indents were performed total. The first 10 were placed to determine optimal load function and maximum depth. Six and seven were then placed on the unoxidized and oxidized samples, respectively, to compare mechanical response of the samples. In each case, indents were placed on the "head" and the "rear" of each of three polymer buffaloes per unoxidized/oxidized group. $\times 10^{2}$

Different load functions were required for these materials. For the stiffer, oxidized polymer, a two minute stress relaxation hold was generally sufficient to dissipate viscoelastic energy and was thus employed. For the less-stiff polymer, a response of sink-in or material compression was inevitable with this tip (which led to artificial "stiffening" during the stress relaxation hold). The next larger spherical tip, $r=100 \mu \mathrm{m}$, would have had a greater radius than the buffalo pattern, so better results were obtained by reducing the hold time to two seconds to avoid sink-in / compression. The same indentation depth was used for each test (150 nm), and was so chosen because the much stiffer substrate $(\sim 70 \mathrm{GPa})$ is more likely to influence the stiffness at greater depths. We noticed that indent depths of even $300 \mathrm{~nm}$, ostensibly only $10 \%$ of film stiffness, produced a modulus of nearly $20 \mathrm{GPa}$ for the oxidized polymer. This value is an order of magnitude greater than at $150 \mathrm{~nm}$ depth and thus strong evidence for a substrate effect. Indents shallower than $150 \mathrm{~nm}$ become quite challenging for the unoxidized polymer due to the extremely low indentation forces. Lastly, both unoxidized and oxidized polymers demonstrate some adhesion during the unloading stage. The estimated indentation modulus may be artificially high because of this effect. Further analyses (nano-JKR) method can be pursued if requested to provide a more exact estimate.

\section{Analytic method}

The unloading curves were analyzed using an Oliver-Pharr approach using Hysitron Triboscan software. Using Oliver-Pharr, the nanoindentation modulus $\left(\mathrm{E}_{\mathrm{r}}\right)$ is given as:

Equation 1: $\quad E_{r}=\frac{\sqrt{\pi}}{2} \frac{S}{\sqrt{A_{c}}}$ 
Here, $\mathrm{S}$ is the contact stiffness, and is measured as the slope of the initial part of the unloading curve. The $95-20 \%$ portion of the unloading curve is fit with a power curve, where the derivative of the curve at the start of unloading calculates stiffness. $A_{c}$ is the contact area, which is a function of contact depth, and is calibrated on a silica reference standard.

Note, $\mathrm{E}_{\mathrm{r}}$ is the reduced modulus, and is the modulus of the specimen-indenter combination.

The indentation modulus $\left(\mathrm{E}_{\mathrm{i}}\right)$ is given as:

Equation 2: $\quad E_{i}=\left(\frac{1}{E_{r}}-\frac{1-v_{t i p}^{2}}{E_{\text {tip }}}\right)^{-1}=\frac{E_{s}}{1-v_{s}^{2}}$

For this equation, $v$ is the Poisson's ratio, and "s" are the elastic properties of the sample. The Poisson's ratio and elastic modulus for the tip are assumed to be 0.07 and $1140 \mathrm{GPa}$, respectively. The indentation modulus is not an absolute property for the sample, but is a function of the sample modulus and the sample Poisson's ratio. If the Poisson's ratio is known for the sample, the sample modulus may be calculated using the relation in Equation 2 (note: these samples adhere to the tip during unloading, which will likely cause some amount of error in the indentation estimates).

The hardness is given as:

Equation 3: $\quad H=\frac{P_{\max }}{A_{c}}$

$P_{\max }$ is the max load.

Unoxidized material

\begin{tabular}{|c|c|c|c|c|c|c|c|c|c|}
\hline & $\begin{array}{l}\text { Er all } \\
\text { indents } \\
\text { (MPa) }\end{array}$ & $\begin{array}{l}\text { Er } \\
\text { "head" } \\
\text { (MPa) }\end{array}$ & $\begin{array}{l}\text { Er } \\
\text { "rear" } \\
\text { (MPa) }\end{array}$ & $\begin{array}{l}\text { Ei all } \\
\text { indents } \\
\text { (MPa) }\end{array}$ & $\begin{array}{l}\text { Ei } \\
\text { "head" } \\
\text { (MPa) }\end{array}$ & $\begin{array}{l}\text { Ei } \\
\text { "rear" } \\
\text { (MPa) }\end{array}$ & $\begin{array}{l}\mathrm{H} \text { all } \\
(\mathrm{MPa})\end{array}$ & $\begin{array}{l}\text { H } \\
\text { "head" } \\
\text { (MPa) }\end{array}$ & $\begin{array}{l}\mathrm{H} \\
\text { "rear" } \\
(\mathrm{MPa})\end{array}$ \\
\hline & 75.17 & 81.21 & 69.12 & 75.17 & 81.22 & 69.13 & & 6.48 & 6.26 \\
\hline $\begin{array}{l}\text { Standard } \\
\text { Deviation }\end{array}$ & 18.42 & 15.86 & 22.07 & 18.42 & 15.86 & 22.07 & 0.38 & 0.32 & 0.48 \\
\hline
\end{tabular}

Oxidized material

\begin{tabular}{|l|l|l|l|l|l|l|l|l|l|}
\hline & $\begin{array}{l}\text { Er all } \\
\text { indents } \\
(\mathrm{GPa})\end{array}$ & $\begin{array}{l}\text { Er } \\
\text { "head" } \\
(\mathrm{GPa})\end{array}$ & $\begin{array}{l}\text { Er } \\
\text { "rear" } \\
(\mathrm{GPa})\end{array}$ & $\begin{array}{l}\text { Ei all } \\
\text { indents } \\
(\mathrm{GPa})\end{array}$ & $\begin{array}{l}\text { Ei } \\
\text { "head" } \\
(\mathrm{GPa})\end{array}$ & $\begin{array}{l}\text { Ei } \\
\text { "rear" } \\
(\mathrm{GPa})\end{array}$ & $\begin{array}{l}\mathrm{H} \text { all } \\
(\mathrm{GPa})\end{array}$ & $\begin{array}{l}\mathrm{H} \\
\text { "head" } \\
(\mathrm{GPa})\end{array}$ & $\begin{array}{l}\mathrm{H} \\
\text { "rear" } \\
(\mathrm{GPa})\end{array}$ \\
\hline Average & 5.83 & 4.28 & 6.99 & 5.87 & 4.30 & 7.04 & 0.11 & 0.07 & 0.14 \\
\hline $\begin{array}{l}\text { Standard } \\
\text { Deviation }\end{array}$ & 2.77 & 3.29 & 1.97 & 2.79 & 3.32 & 1.99 & 0.08 & 0.07 & 0.07 \\
\hline
\end{tabular}

The unoxidized polymer and oxidized polymer have markedly different nanoindentation moduli; the average unoxidized polymer indentation modulus is measured to be $75.17 \mathrm{MPa}$, while the oxidized polymer indentation modulus is almost two orders of magnitude larger at $5.87 \mathrm{GPa}$. 